\title{
Broadband anti-reflection coating for the meter class Dark Energy Spectroscopic Instrument lenses
}

Charles Kennemore, Debi Archer, Russ Barbaria, Joe Bertolina, Rick Dix, et al.

Charles Kennemore, Debi Archer, Russ Barbaria, Joe Bertolina, Rick Dix, Katy Dodson, Sharon Doi-Okray, Aaron Gallon, Bill Sweet, Timothy N. Miller, Robert W. Besuner, Michael E. Levi, Michael Lampton, Pat Jelinsky, Jerry Edelstein, David J. Schlegel, Jeremy McCauley, Henry Heetderks, Michael J. Sholl, Peter Doel, David Brooks, Stephen Kent, "Broadband antireflection coating for the meter class Dark Energy Spectroscopic Instrument lenses," Proc. SPIE 10706, Advances in Optical and Mechanical Technologies for Telescopes and Instrumentation III, 107061S (10 July 2018); doi:

$10.1117 / 12.2313006$

Event: SPIE Astronomical Telescopes + Instrumentation, 2018, Austin, Texas, United States 


\title{
Broadband anti-reflection coating for the meter class Dark Energy Spectroscopic Instrument lenses
}

Charles Kennemore III ${ }^{\mathrm{a}}$, Debi Archer ${ }^{\mathrm{a}}$, Russ Barbaria ${ }^{\mathrm{a}}$, Joe Bertolina ${ }^{\mathrm{a}}$, Rick Dix ${ }^{\mathrm{a}}$, Katy Dodson $^{\mathrm{a}}$, Sharon Doi-Okray ${ }^{\mathrm{a}}$, Aaron Gallon ${ }^{\mathrm{a}}$, Bill Sweet ${ }^{\mathrm{a}}$, Timothy N. Miller ${ }^{\mathrm{b}}$, Robert W. Besuner ${ }^{\mathrm{b}}$, Michael E. Levi ${ }^{\mathrm{b}}$, Michael Lampton ${ }^{\mathrm{b}}$, Pat Jelinsky ${ }^{\mathrm{b}}$, Jerry Edelstein ${ }^{\mathrm{b}}$, David J. Schlegel ${ }^{\mathrm{b}}$, Jeremy McCauley ${ }^{\mathrm{b}}$, Henry Heetderks ${ }^{\mathrm{b}}$, Michael Sholl ${ }^{\mathrm{b}, \mathrm{c}}$, Peter Doel ${ }^{\mathrm{d}}$, David Brooks ${ }^{\mathrm{d}}$, Stephen Kent ${ }^{\mathrm{e}}$, for the DESI Collaboration

${ }^{\mathrm{a}}$ Viavi Solutions, 1402 Mariner Way, Santa Rosa, CA, USA 95407; ${ }^{\mathrm{b}}$ Lawrence Berkeley National Laboratory, 1 Cyclotron Rd, Berkeley, CA, USA 94720; 'SpaceX Inc., 1 Rocket Road, Hawthorne, CA, USA 90250; ${ }^{\mathrm{d}}$ Department of Physics \& Astronomy, University College London, Gower St, London WC1E 6BT, United Kingdom; ${ }^{\mathrm{e}}$ Fermi National Accelerator Laboratory, PO Box 500, Batavia, IL, USA 60510

\begin{abstract}
The Dark Energy Spectroscopic Instrument (DESI), currently under construction, will be used to measure the expansion history of the Universe using the Baryon Acoustic Oscillation technique. The spectra of 35 million galaxies and quasars over $14000 \mathrm{sq} \mathrm{deg}$ will be measured during the life of the experiment. A new prime focus corrector for the KPNO Mayall telescope will deliver light to 5000 fiber optic positioners. The fibers, in turn, feed ten broad-band spectrographs. We will describe the broadband AR coating $(360 \mathrm{~nm}$ to $980 \mathrm{~nm}$ ) that was applied to the lenses of the camera system for DESI using ion assisted deposition techniques in a $3 \mathrm{~m}$ coating chamber. The camera has 6 lenses ranging in diameter from 0.8 $\mathrm{m}$ to $1.14 \mathrm{~m}$, weighing from $84 \mathrm{~kg}$ to $237 \mathrm{~kg}$ and made from fused silica or BK7. The size and shape of the surfaces provided challenges in design, uniformity control, handling, tooling and process control. Single surface average transmission and minimum transmission met requirements. The varied optical surfaces and angle of incidence considerations meant the uniformity of the coating was of prime concern. The surface radius of curvature (ROC) for the 12 surfaces ranged from nearly flat to a ROC of $611 \mathrm{~mm}$ and a sag of $140 \mathrm{~mm}$. One lens surface has an angle of incidence variation from normal incidence to $40^{\circ}$. Creating a design with a larger than required bandwidth to compensate for the non-uniformity and angle variation created the ability to reduce the required coating uniformity across the lens and a single design to be used for all common substrate surfaces. While a perfectly uniform coating is often the goal it is usually not practicable or cost effective for highly curved surfaces. The coating chamber geometry allowed multiple radial positions of the deposition sources as well as substrate height variability. Using these two variables we were able to avoid using any masking to achieve the uniformity required to meet radial and angle performance goals. Very broadband AR coatings usually have several very thin and optically important layers. The DESI coating design has layers approaching $3 \mathrm{~nm}$ in thickness. Having sensitive thin layers in the design meant controlling layer thickness and azimuthal variation were critical to manufacturing repeatability. Through use of strategically placed quartz crystal monitors combined with stable deposition plumes, the manufacturing variability was reduced to acceptable levels. Low deposition rates and higher rotation rates also provided some stability to azimuthal variation.
\end{abstract}

Keywords: optical coating, lens, DESI, anti-reflection

\section{INTRODUCTION}

The design of broadband anti-reflection (BBAR) coatings have been studied for many years ${ }^{1,2,3,4}$ and yet even with new insights, it is still a challenging task to produce the performance as designed. The number of layers increases as the wavelength bandwidth increases and several of the layers become optically very thin, adding complexity and increasing error possibilities in the coating process. Modern controls and systems have allowed this complexity to be more tightly controlled for many applications, but as the surfaces start becoming larger and more curved the complexity rises again adding further constraints to coating geometry, uniformity, and handling. The coating requirements for the DESI Project ${ }^{6}$ bring all these complicating factors into one optical system - wide bandwidth, low reflectance, large size, coating 
uniformity concerns, and handling hundred-kilogram optics. Usually, coating meter size optics means coating one or two mirrors or windows but the DESI project is a multiple lens corrector design ${ }^{7}$. The six DESI lenses range in size from 0.8 $\mathrm{m}$ to $1.14 \mathrm{~m}$ and present 12 unique surfaces that require the same AR performance. The full task can be glimpsed in Figure 1 which shows all the lens to scale arranged as used in the final corrector assembly. The surfaces run the full gamut from the deeply convex $\mathrm{C} 4$ to the deeply concave $\mathrm{C} 2$ with the $\mathrm{ADC}$ lenses being N-BK7 instead of fused silica like lens $\mathrm{C} 1-\mathrm{C} 4$.

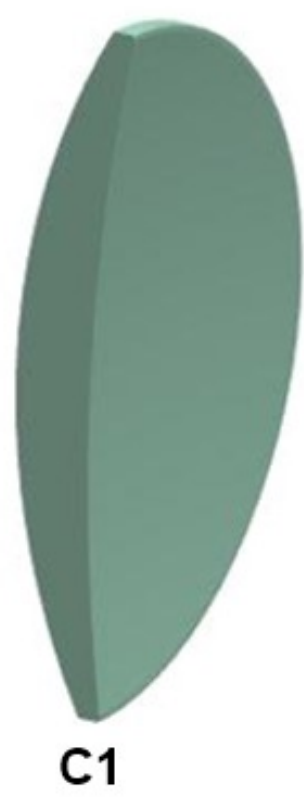

C1 - C4: fused silica ADC1-2: N-BK7

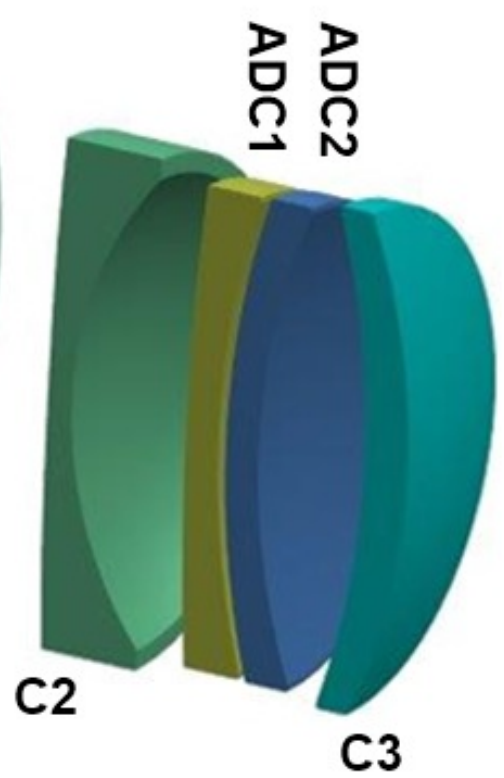

C3

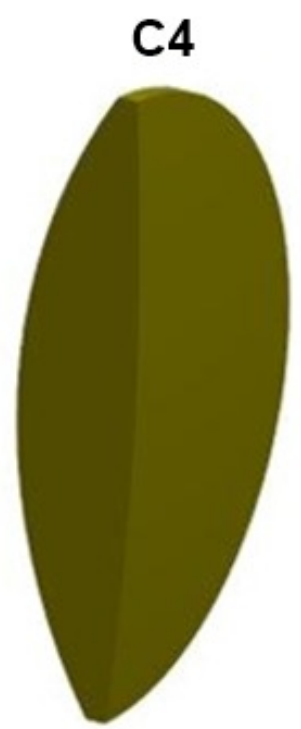

Figure 1. The six lenses of the DESI lenses system arranged in cross section as in the final assembly. The two ADC lenses are N-BK7 with the other 4 lenses being made of fused silica. All surfaces require AR coating.

The single surface requirements for each lens surface are given in Table 1. Complicating the transmission requirements is the range of angles across some of the lenses. The range of angles of incidence on each lens surface also varies across the clear aperture. The average angle as a function of radius is shown in Figure 2. Three of the surfaces have angle ranges over $20^{\circ}$ with the highest angle approaching $40^{\circ}$. The band average transmission requirement was met for each surface, but the minimum transmission specification was not met on three of the surfaces, C1A, C4A, and ADC2B. The lowest measured transmission performances across the clear aperture achieved for each surface are given in Table 2. To gauge the system impact of the measured transmission of each surface on the full 6 lens system the estimated 12 surface transmission is calculated at three radial positions - center of each surface, mid-radius of each surface, and at the clear aperture of each surface - to give a measure of the uniformity of the performance across the full clear aperture. The data accounts for radial non-uniformity and angle at the point of measurement. Assuming the required system average transmission is the $12^{\text {th }}$ power of the required single surface transmission then system average transmission requirement is found to be greater than $84.4 \%$ and the minimum transmission is to be greater than $78.5 \%$. This data is shown in Figure 3 and it can be seen the system performance will comfortably surpass the extrapolated required transmission. Table 3 shows the numerical averages plotted in Figure 3. The system performance exceeds the requirements by a healthy margin even with the three surfaces that have a lower than desired minimum transmission. For each surrogate coating run and lens coating run, a few witnesses were tested to assure compliance with the durability requirements. All 
runs throughout the 2-year program passed the durability testing showing no degradation either physically or spectrally after the durability sequence was completed.

Table 1. Single surface coating requirements for DESI lenses

\begin{tabular}{|l|c|}
\hline \multicolumn{1}{|c|}{ Requirement } & Specification \\
\hline Single Surface Average Transmission $-360 \mathrm{~nm}$ to $980 \mathrm{~nm}$ & $98.5 \%$ \\
\hline Single Surface Minimum Transmission - $360 \mathrm{~nm}$ to $980 \mathrm{~nm}$ & $98.0 \%$ \\
\hline Coating Quality & $80-50$ \\
\hline Durability; Humidity & MIL-C-48497A, humidity \\
\hline Durability: adhesion & MIL-C-48497A, quick tape \\
\hline Durability: abrasion & MIL-C-48497A, moderate abrasion \\
\hline Durability: Solubility and Cleanability & MIL-C-48497A, acetone and alcohol \\
\hline Survivable temperature range & $-20^{\circ} \mathrm{C}$ to $+60^{\circ} \mathrm{C}$ \\
\hline
\end{tabular}

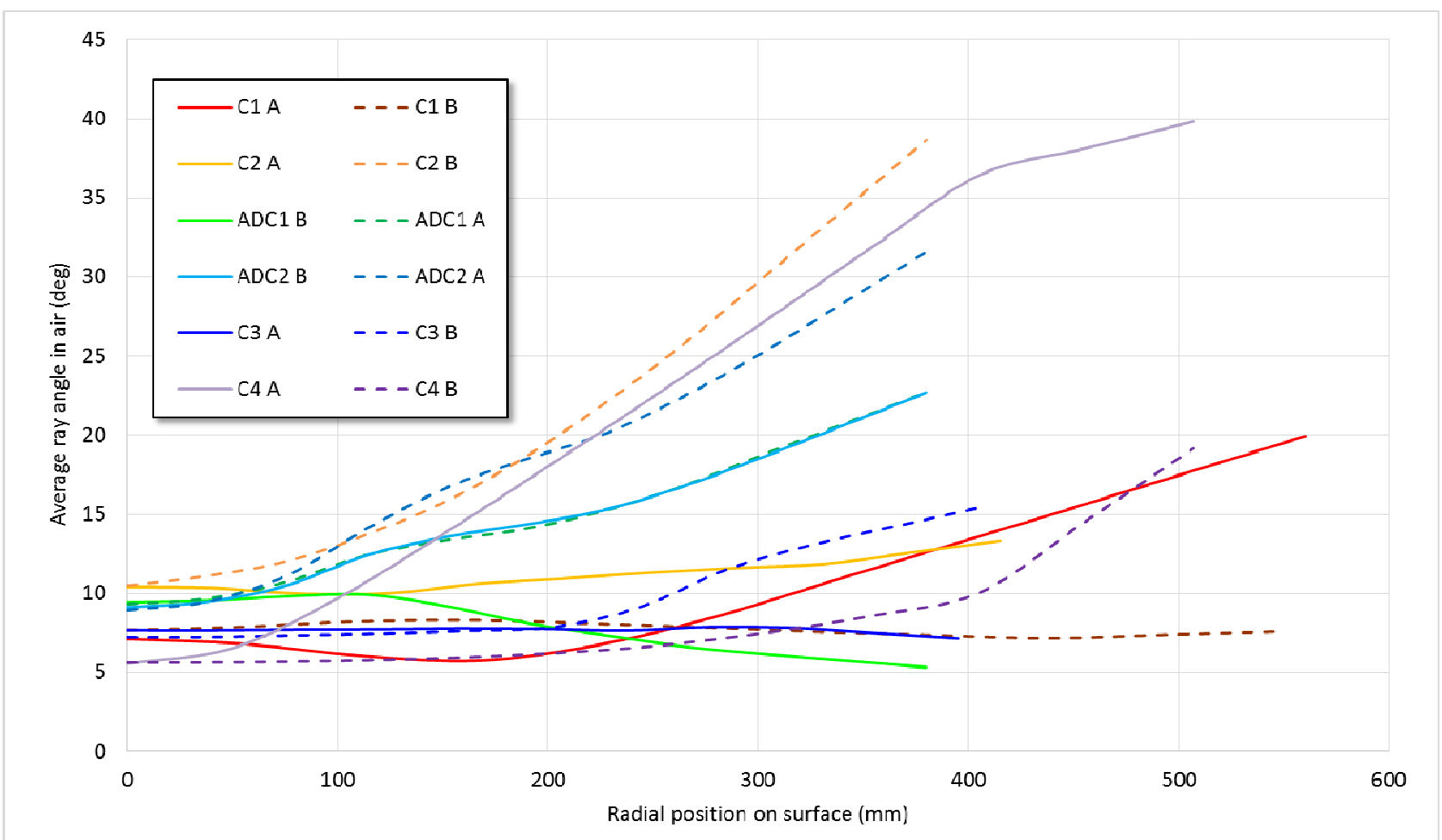

Figure 2. The average angle of incidence for each DESI lens surface as a function of radius. 
Table 2. Measured transmission performance for each lens surface at the radius of minimum performance.

\begin{tabular}{|c|c|c|c|}
\hline Lens & Surface & $\begin{array}{c}\text { Lowest } \\
\text { average } \\
\text { transmission } \\
\text { across radius } \\
\mathbf{( \% )}\end{array}$ & $\begin{array}{c}\text { Lowest } \\
\text { minimum } \\
\text { transmission } \\
\text { across } \\
\text { radius (\%) }\end{array}$ \\
\hline specification & - & 98.5 & 98.0 \\
\hline C1 & A & 98.9 & $\mathbf{9 7 . 9}$ \\
\hline C1 & B & 99.0 & 98.1 \\
\hline C2 & A & 99.2 & 98.9 \\
\hline C2 & B & 99.1 & 98.7 \\
\hline C3 & A & 99.3 & 98.4 \\
\hline C3 & B & 99.1 & 98.8 \\
\hline C4 & A & 98.7 & $\mathbf{9 1 . 7}$ \\
\hline C4 & B & 99.1 & 98.0 \\
\hline ADC1 & A & 99.0 & 98.6 \\
\hline ADC1 & B & 99.2 & 98.8 \\
\hline ADC2 & A & 99.1 & 98.3 \\
\hline ADC2 & B & 98.9 & $\mathbf{9 7 . 7}$ \\
\hline
\end{tabular}

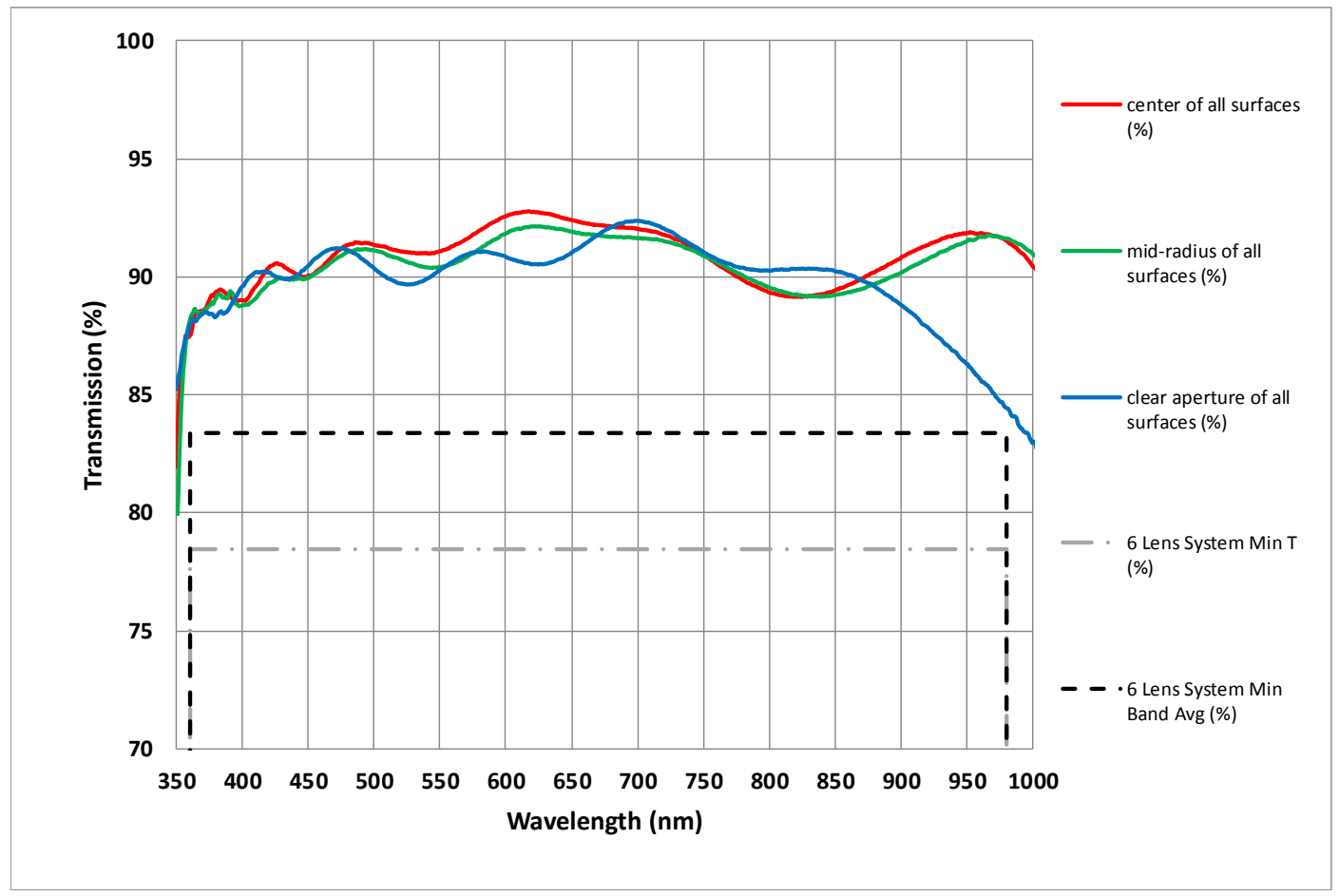

Figure 3. Estimated total transmission of the 12 surfaces of the 6-lens system using values at the center, mid-radius and clear aperture of each lens. The required system minimum and band average minimum is calculated as the 12 th power of 0.98 and 0.985 respectively. The system transmission of the coated surface exceeds the band average minimum by $7 \%$ and the required system minimum by almost $15 \%$. 
Table 3. Estimated 6 lenses system performance of DESI at three different radii.

\begin{tabular}{|l|c|c|}
\hline Component & $\begin{array}{c}\text { Average } \\
\text { Transmission - } \\
\mathbf{3 6 0} \mathbf{~ n m ~ - ~ 9 8 0} \\
\mathbf{n m ~ ( \% )}\end{array}$ & $\begin{array}{c}\text { Minimum } \\
\text { Transmission - } \\
\mathbf{3 6 0} \mathbf{~ n m ~ - ~ 9 8 0 ~} \\
\mathbf{n m ~ ( \% )}\end{array}$ \\
\hline 6 lens system & 83.4 & 78.5 \\
\hline center values & 90.9 & 87.5 \\
\hline mid-radius values & 90.6 & 88.1 \\
\hline at clear aperture values & 90.0 & 84.4 \\
\hline
\end{tabular}

In achieving the results just reviewed Viavi developed and fabricated tooling to safely handle the lenses at all phases of the coating process. To minimize set up time between coating different lens surfaces and to keep costs as low as possible the tooling design concept created centralized pieces to be common to all the lens surfaces using smaller elements to adapt the common piece to each new surface shape. With the wide range of lens surface curvatures, the adaptability of Viavi's coating chamber geometry enables acceptable uniformities across the lens surfaces without resorting to the use of masking. Once the tooling was developed and uniformities modeled, witnesses arrayed on surrogates for each surface were used to confirm the coating was compliant with requirements prior to coating each lens surface.

\section{LENS HANDLING AND TOOLING}

Each lens needs to be removed from the shipping crate, cleaned, coated, and put back into the shipping crate. The full process requires cleaning the lens twice, rotating it to have either side up or down as needed, moved into a coating chamber, heated to deposition temperatures, cooled back down, removed from the chamber and inspected. This had to be done twice for each lens - once for each side - and all inside Viavi's Class 100 clean room. The tooling must also be adjustable for all 6 lenses. The two largest challenges were safely rotating each lens and assuring the thermal cycling did not create expansion mismatches between the two different glass types and the common support tooling. Viavi developed cleaning rings and supports allowing the lenses to be easily positioned in any orientation for cleaning, inspection, or transfer to other tooling. The cleaning tooling is also resistant to the cleaning solvents used in cleaning each lens surface. To minimize the thermal mismatch between the lens and tooling during coating operations, the coating tooling was constructed of Invar and length tuned to allow both the low expansion fused silica and higher expansion BK7 lenses to be safely held during thermal cycles while still maintaining the required clear aperture. Two rings were used as the common load support for all the lenses: one for cleaning and one for coating. This approach simplified interfacing to lifting equipment and all other mounts. Each lens then required simple adapters to be installed on the rings to handle the different diameters and surface shapes. The tooling was analyzed using FEA to assure loads and stresses were within safe limits. A final physical test of each tooling was done at 1.5 times maximum weight before use with any lens. The load testing of the Coating Ring is shown on the left side of Figure 4.

Part of the handling operations was to develop a set of procedures precisely stepping through each operation as the lens traveled from the shipping crate to the coating chamber and back again. The Viavi team designed the tooling and developed the procedures in parallel, aligning design intent with handling process reality to assure the final tooling design was useable with minimal test/adjustment cycles. The resulting 550-step procedure detailing handling operations for each lens surface as it progresses through the coating process allows a small, experienced team to safely process each lens twice as both sides are coated. A key position on the team is the person whose sole job is to assure each step on the handling procedure is followed as intended, physically check off each step as completed, and verbally read out each procedure step as needed. After the tooling was fabricated, a metal mass surrogate of the heaviest lens was used to walk through each step of the process, fine tuning process details and assuring the tooling functioned as intended. The right side of Figure 4 shows the mass surrogate at $1 \mathrm{X}$ weight during a full-scale walk-through of the procedures. Once the procedures and practice were completed then a lens could be processed. The detailed procedure, experienced personnel, and mass surrogate practice allowed each lens to be safely handled twice as each of the 12 surfaces were coated. The C4 lens with the A side down in the coating chamber is shown in Figure 5. 

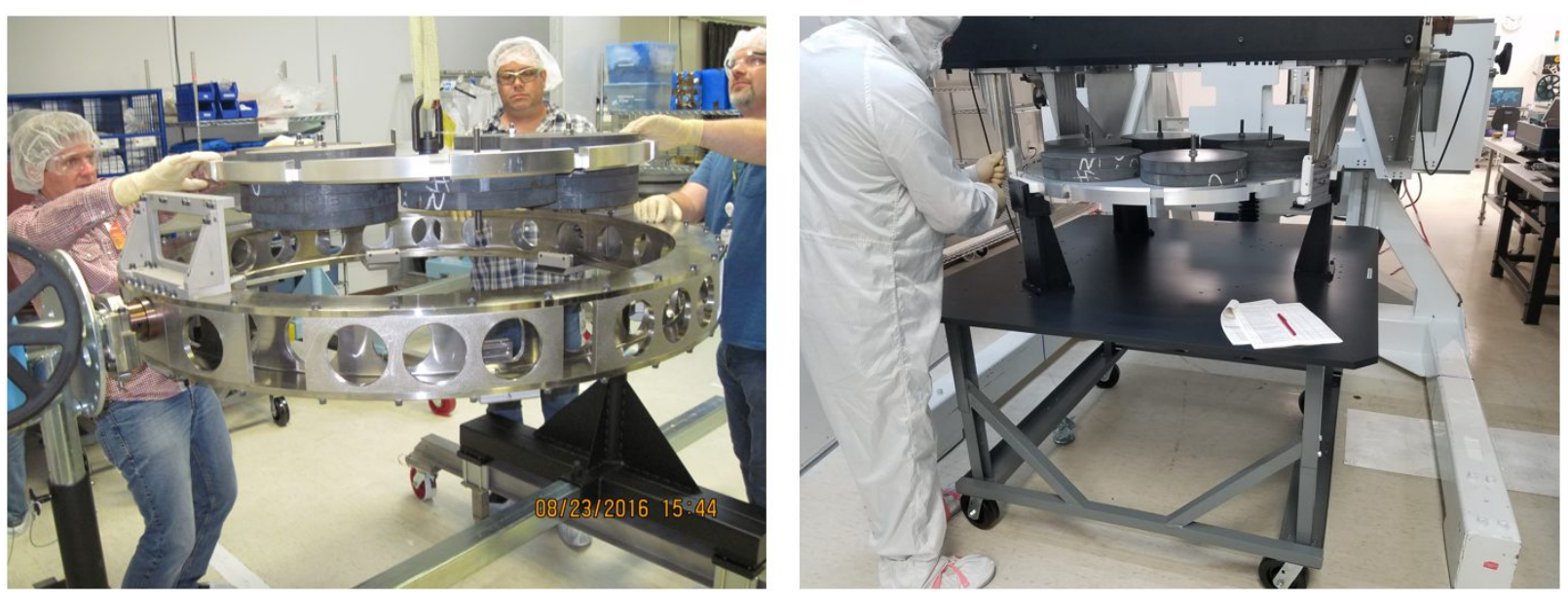

Figure 4. Load testing coating fixture to 1.5 times maximum lens weight using metal mass surrogate is shown on left. Weights were added to increase weight as needed. Testing was completed at $431 \mathrm{~kg}, 1.5$ times the weight of the heaviest lens, C4. Practice and test procedures with full weight metal C4 surrogate is shown on right. The operation shown is practice moving the lens to a mobile transfer table from the shipping crate.

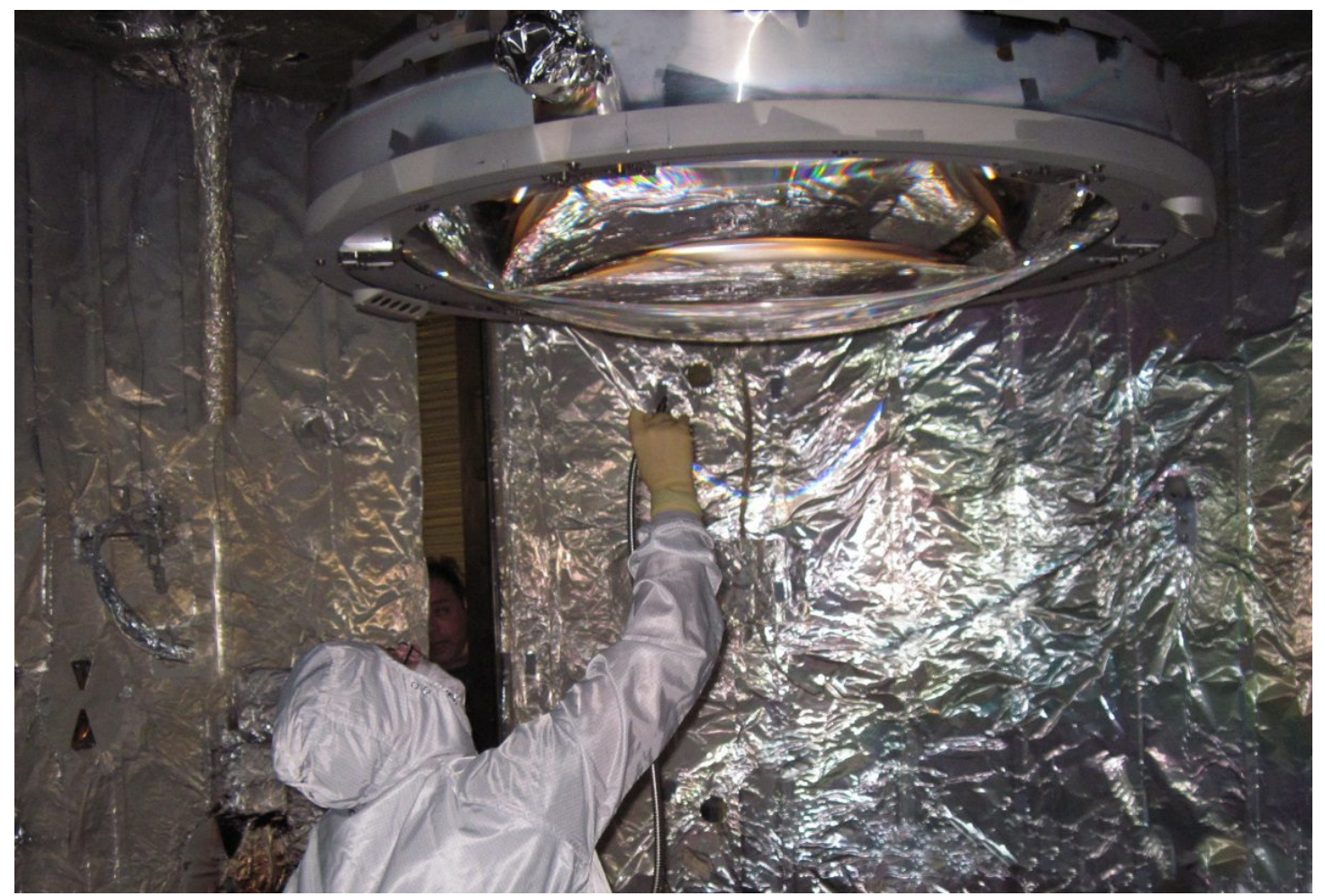

Figure 5. C4 lens in Coating Ring in the chamber at final inspection before starting chamber evacuation.

\section{CHAMBER GEOMETRY AND COATING NON-UNIFORMITY}

The diameter and wide range of curvatures of the 6 lenses present the challenge in that no single geometry gives the best solution to optimizing the coating uniformity on all 12 surfaces. Creating custom masking built around a single coating geometry was a solution Viavi rejected as too costly and complicated. Viavi's coating chamber provides the ability to adjust the radial location of coating sources while coating support tooling allowed for relatively large height changes as needed. Using coating uniformity modeling software, Viavi created three coating source configurations and eight height 
variations that provided an optimized coating non-uniformity for each lens surface without resorting to complex masking schemes. Figure 6 shows the modeled coating uniformity on each surface as a function of radius. Actual results followed the model well. With the expected non-uniformity established, the coating design could be adjusted to maintain performance while the center wavelength shifted as a function of radius.

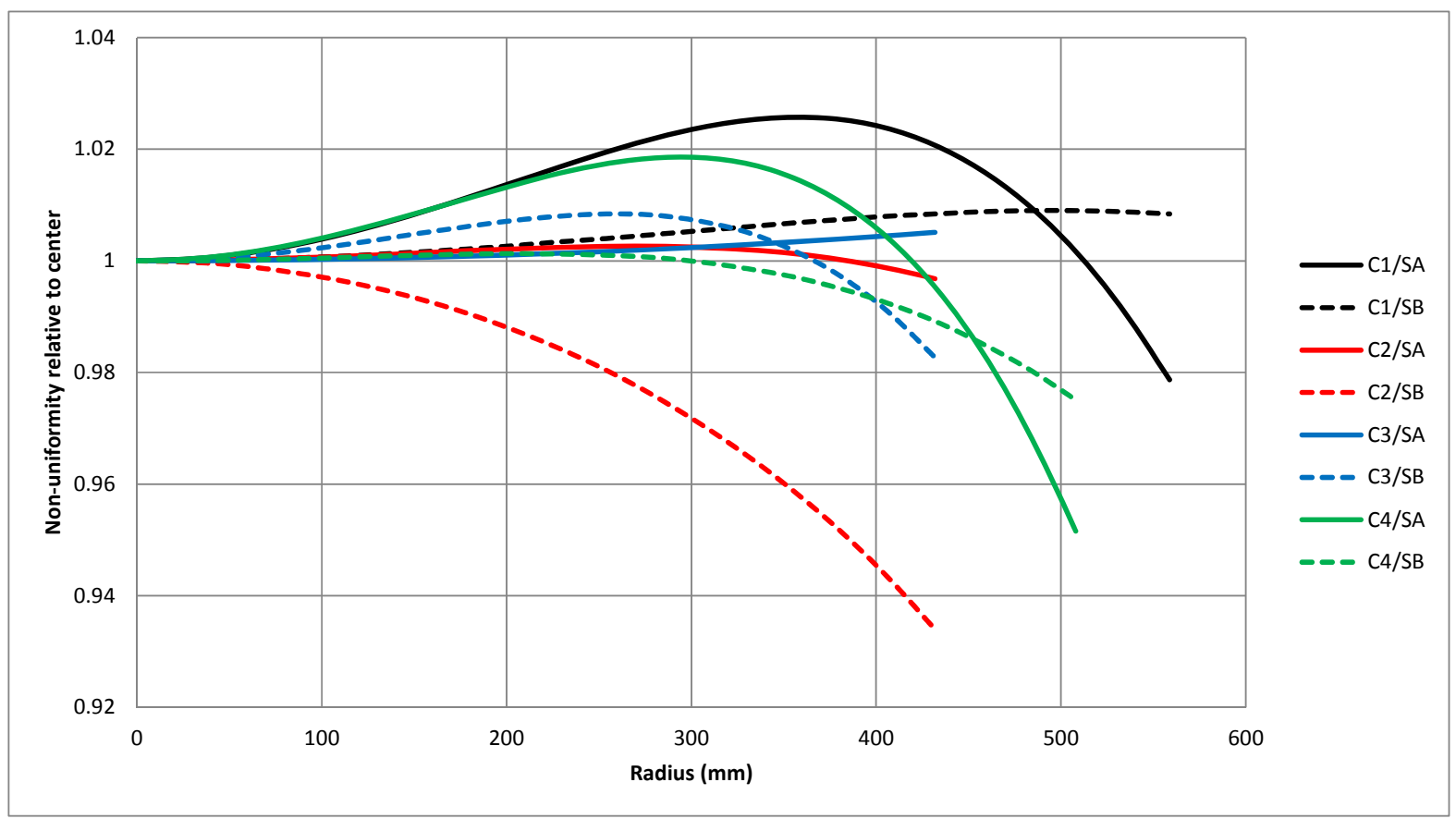

Figure 6. Modeled coating thickness uniformity relative to the center of the lens of the 12 DESI lenses surfaces with each surface having the best coating geometry

The design required the use of very thin layers in the coating which can be difficult to both monitor and reproduce on large substrates. Positioning monitors to closely monitor deposition rates and coating thicknesses allowed the accurate deposition of layers down to $3.5 \mathrm{~nm}$ in thickness. Reliable and uniform deposition of thin layers on large substrates requires close attention to all aspects of deposition geometries. Careful modeling of each chamber configuration for optimal uniformity allowed the best choice of deposition rates, ion flux, rotation rates and monitoring locations. This reduced geometry driven thickness errors to a level that the error induced was negligible compared to expected run to run variabilities. Critical monitoring hardware mounting was designed to be highly repeatable during the conversion between different chamber configurations.

\section{COATING DESIGN}

The coating design must perform with angles of incidence from $5^{\circ}$ to $40^{\circ}$ and a non-uniformity on some surfaces of up to $7 \%$. One possible solution would be to create several different designs with some designs targeted to give good performance for the low non-uniformity and small angle range surfaces and other designs to handle the higher nonuniformity and high angle surfaces. Viavi elected to use a single design for each substrate material. Designing a coating able to accommodate thickness non-uniformity and designing a coating to perform over a many angles of incidence requires a similar approach - make the performance band wider. This allows the coating wavelength centering to shift with the non-uniformity across the surface without sacrificing the overall performance. By necessity, this means the effective band has become wider which means more complexity. For the up to $7 \%$ non-uniformity modeled the effective bandwidth increased from $360 \mathrm{~nm}$ to $980 \mathrm{~nm}$ to a bandwidth of $360 \mathrm{~nm}$ to $1049 \mathrm{~nm}$. A similar approach of designing for high angle ranges is used. As the angle increases the coating wavelength center moves to shorter wavelengths. Other variations besides shift also occur but it is the shift that mainly drives centering concerns. For the $40^{\circ}$ angle, the corresponding blue shift - for the materials used - would be $\sim 8 \%$. A worst-case scenario of a maximum non-uniformity of $7 \%$ (where the coating thickness decreases with radius) and an AOI-induced wavelength shift of $8 \%$ (where the AOI 
increases from near normal to $40^{\circ}$ with radius) the effective band width is increased by $\sim 15 \%$. This now becomes the effective bandwidth for the coating. For DESI specification, the effective increased bandwidth is now $360 \mathrm{~nm}$ to 1127 $\mathrm{nm}$. This new bandwidth became the design target.

In Figure 7 the initial concept designs are shown. Design 1 would allow for the angle shifts or the non-uniformity. Once the final non-uniformity was completed, two more designs were reviewed. By allowing an increase in total coating thickness, Design 2 increased the effective bandwidth of the coating with minor change in the performance across the band. Design 3 was developed to extend the band width and provide increased tolerance to centering errors that were projected to be an area of concern. Design 3 provided a large margin of error for centering, but the decrease in overall transmission decreased performance tolerance and added thickness and complexity to the coating. Based on this analysis Design 2 was the base design for the developmental coating efforts. Design 2 allowed a single design to be used for all the lenses. The design was modified for the two N-BK 7 lenses by matching into that substrate with the same performance criteria applied. Keeping the same design allowed material calibrations to be used for multiple chamber configurations and minimized the number of test runs needed between chamber configurations.

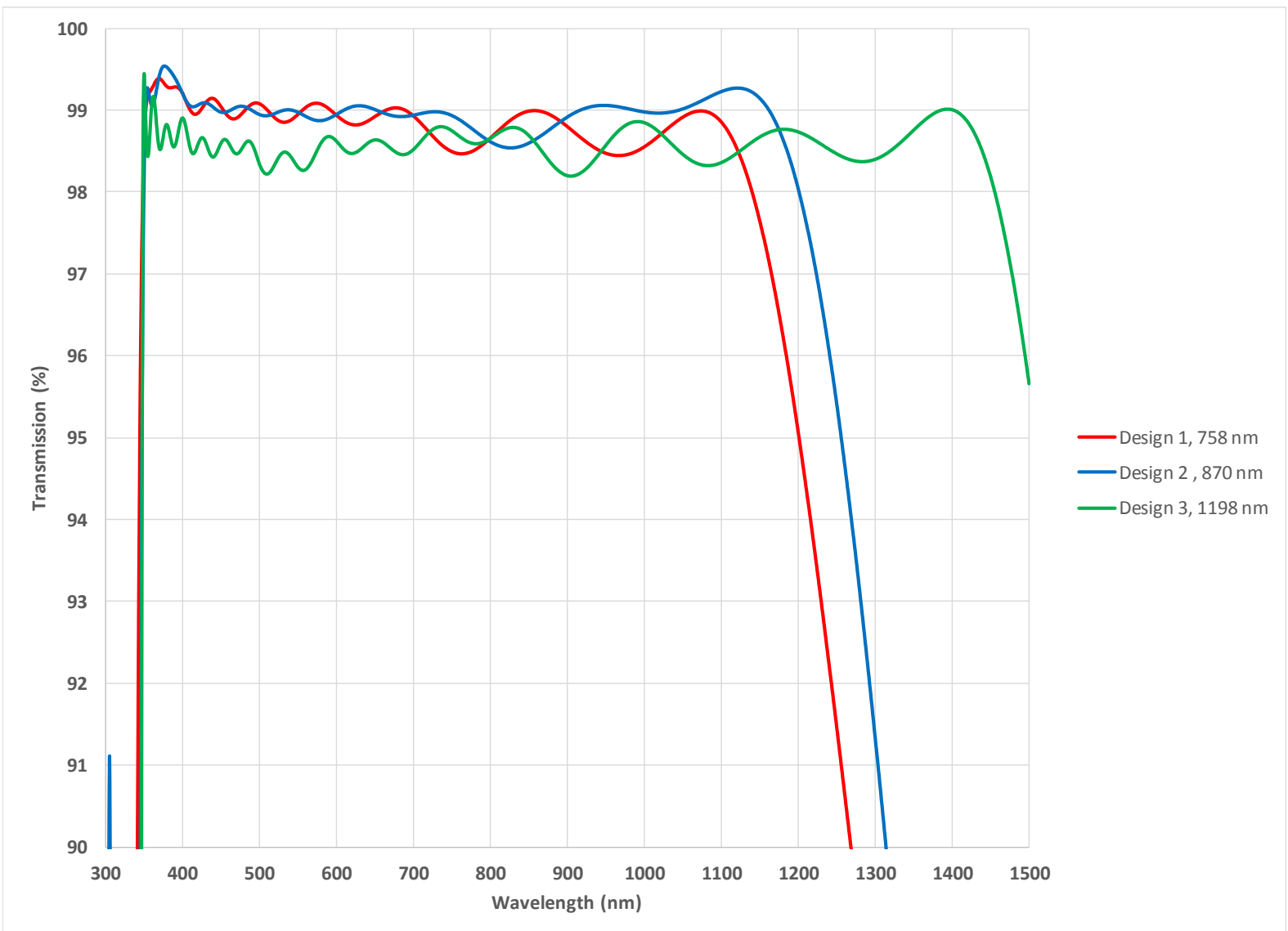

Figure 7. Modeled performance of coating designs analyzed for allowing the anticipated non-uniformities and angle ranges on the lenses to be accommodated. Design 1 was the initial design that accommodated for angle but did not anticipate the extra $7 \%$ of non-uniformity. Design 2 allowed for the modeled non-uniformity to be accommodated. Design 3 was an attempt to increase the coating centering tolerance but was rejected due to the lower transmission performance.

While the simpler design choice is less complex, there are still several very small, optically important layers, that drive the performance especially near the blue and red edges of the coating. The repeatability of coating these thin layers, some as thin as $3.5 \mathrm{~nm}$, is critical to meeting requirements at the band edges. Figure 8 shows the range of layer thicknesses for the two coating designs on fused silica and N-BK7. 


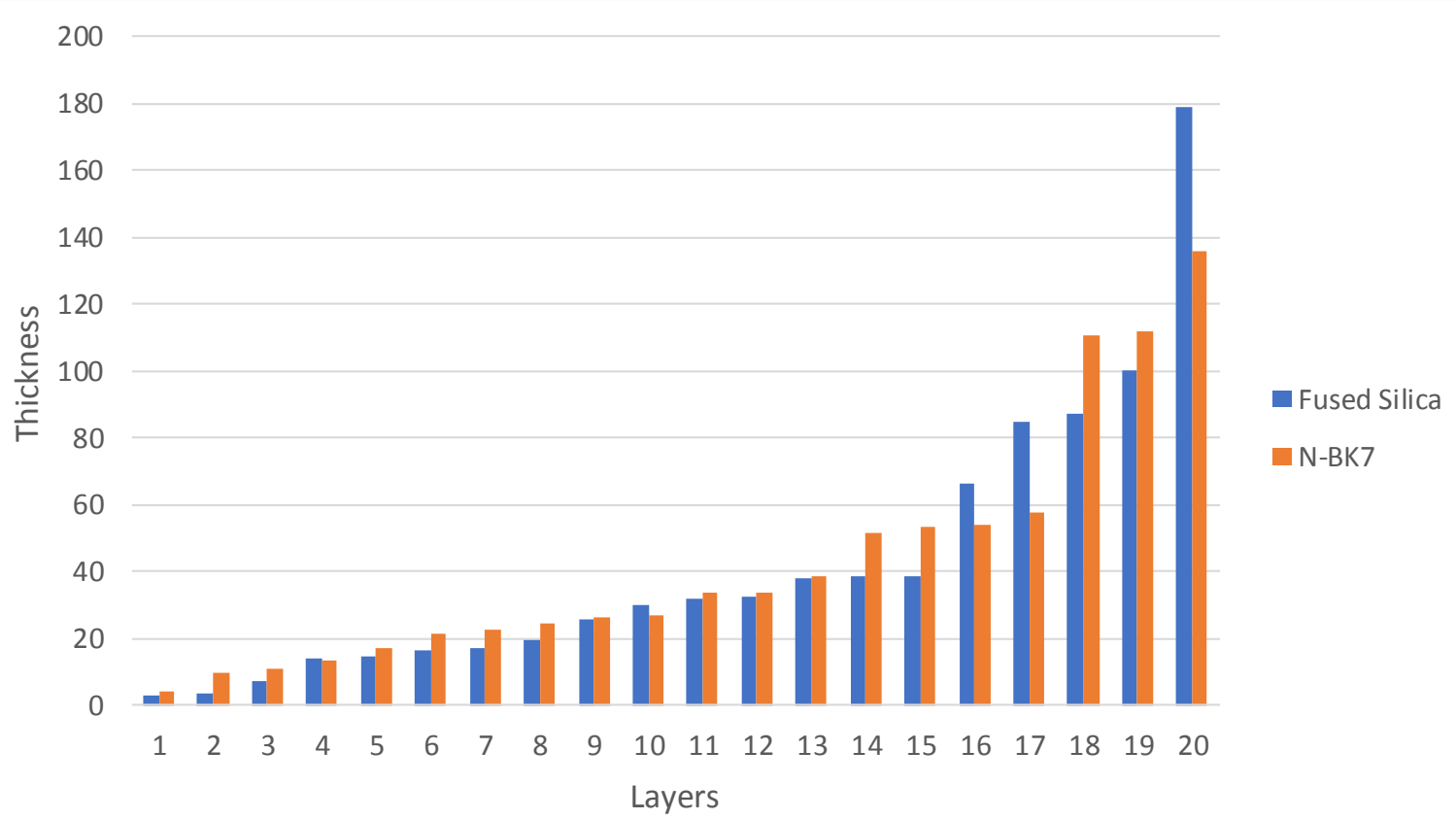

Figure 8. Coating thicknesses of the 20-layer design for fused silica and N-BK7. Fully half of the layers in the coating are less than a quarterwave optical thickness at $400 \mathrm{~nm}$. One fourth of the layers are less than a quarterwave optical thickness at $200 \mathrm{~nm}$.

\section{COATING SURROGATES}

It is important to prove the coating actually meets the intended performance across all angles and across the clear aperture prior to coating a one of a kind optic. While manufacturing a full-scale match of each lens surface that allows some sort of test pieces to be held for coating would be ideal, the costs are significant in such an approach. Viavi designed a surrogate that arrayed 1" diameter witnesses across up to three radii, and supported each witness at the proper angle and radius to form a radial cross section of the actual lens surface. Figures 9 and 10 show CAD renderings of the surrogate for lens C4 Side A and Lens C2 Side B, the two extreme curvature surfaces. Each witness support contains a witness holder that can support three 1" witnesses at the exact angle and radius of a section of the lens. Using three witnesses at each location allowed testing of multiple requirements without sacrificing a witness. For instance, one witness could be used for durability testing while another is measured for spectral performance. It also mitigates the possibility of a witness damaged during post coating testing, and allows a witness to be kept pristine if needed. The witness positions are relatively adjustable, allowing up to 9 radii to be tested for the two $1 \mathrm{~m}$ lenses and 7 radii for the $0.75 \mathrm{~m}$ ones. The surrogate assemblies were attached into the same coating ring fixture as the lenses are coated in to minimize the possible differences in the chamber between a test coating and the actual lens coating. To better represent the actual lens surface the surrogate represents, curved metal surface wedges were fabricated to fill the space between the witness arms. As will be discussed below, the material content of the space between the witness positions can affect the deposition parameters at the witness locations enough to significantly shift the centering of the coating. When installed, the surface wedges effectively form a surface between the witness arms that approximates the surface of the lens being tested. Figure 11 shows a surrogate mounted into the Coating Ring with the surface wedges in place. One of the wedges is outlined in red to more clearly delineate one of the surface wedges in the photo. The surface wedges were rolled to nominally match the surface curvature of the lens surface being tested. During testing of a coating for a specific lens surface the surrogate was mounted in the chamber and the witnesses were tested to confirm the coating met the required specifications. Once a geometry was qualified, the surrogate was removed from the coating ring. The coating ring is now ready for the lens to be installed into the same ring and coated. 


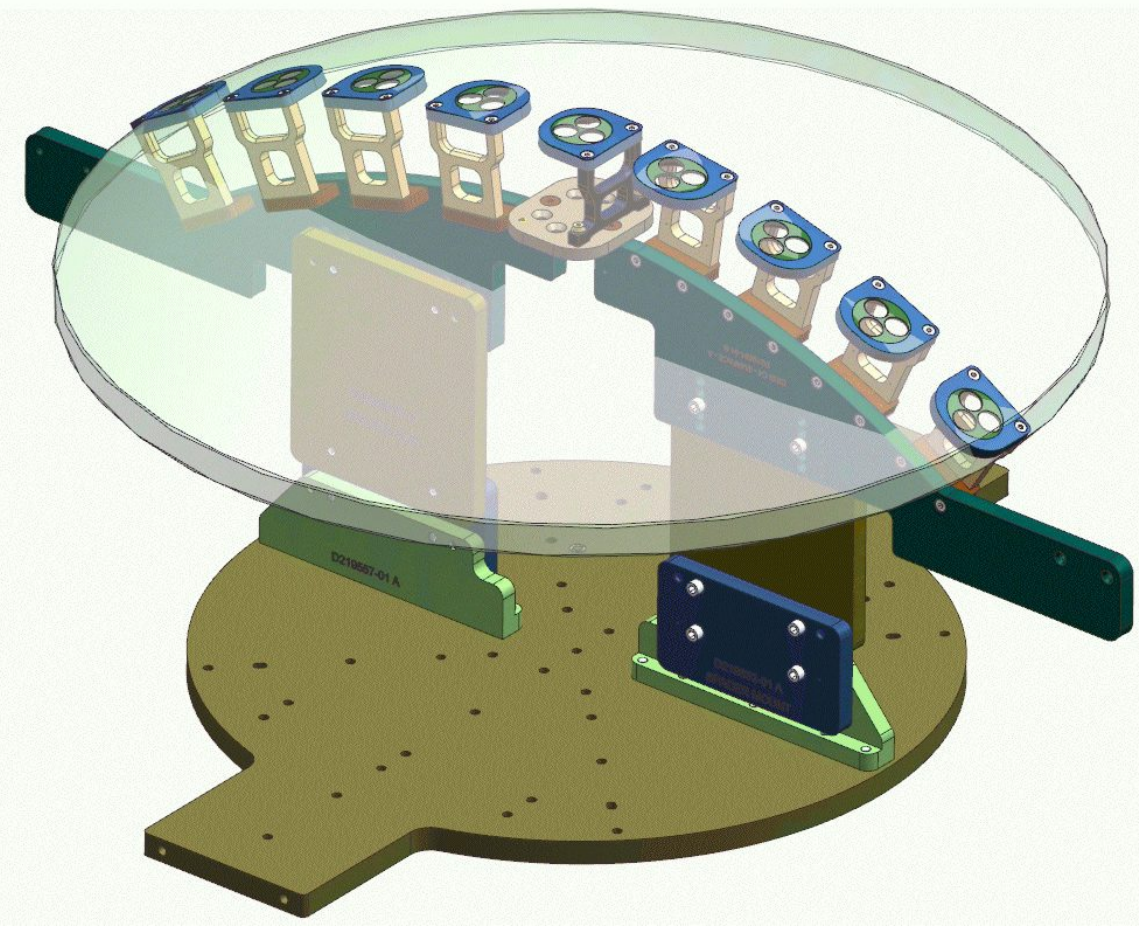

Figure 9. Example of surrogate for the convex side of $\mathrm{C} 1$. A transparent $\mathrm{C} 1$ lens is placed over tooling to show how the witnesses follow the curvature of the lens.

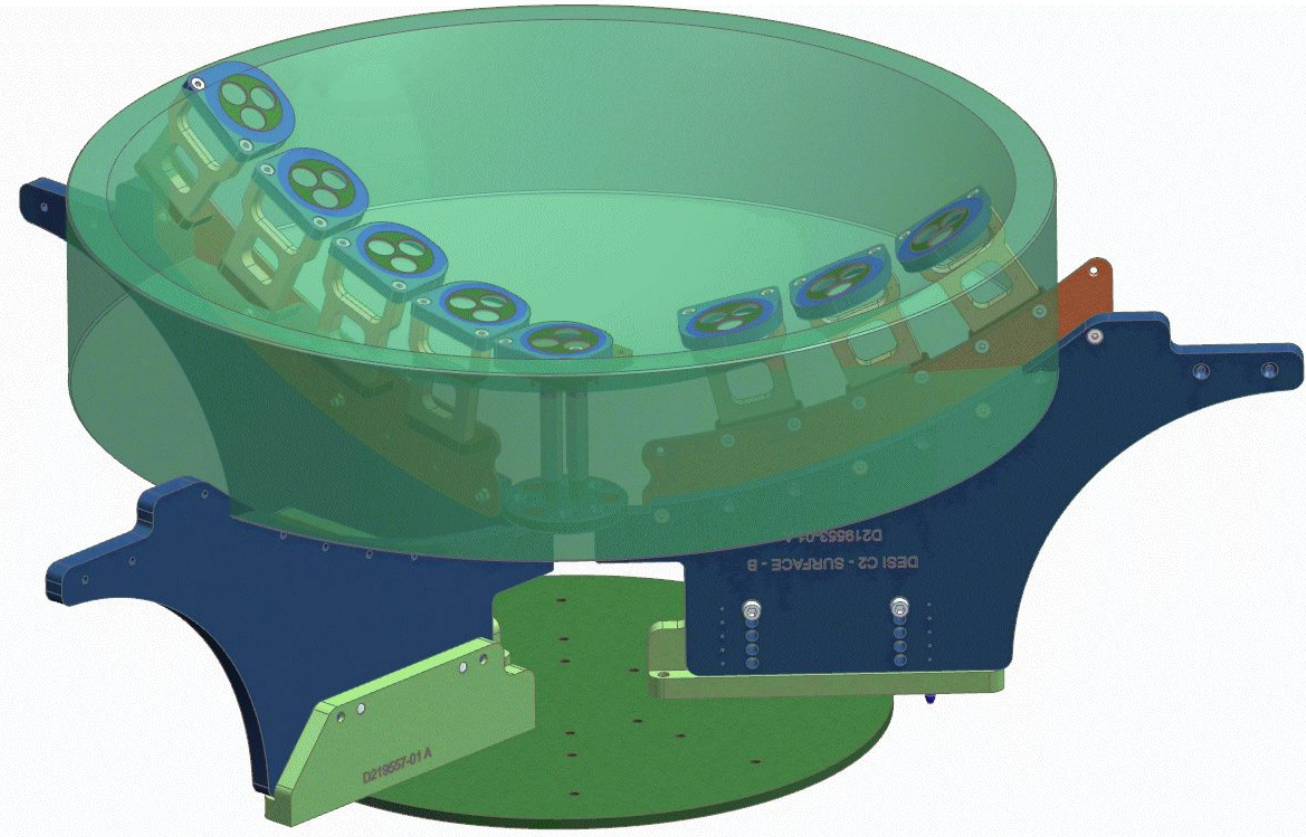

Figure 10. Example of surrogate for the concave side of C2. A transparent C2 lens is placed over tooling to show how the witnesses follow the curvature of the lens. 


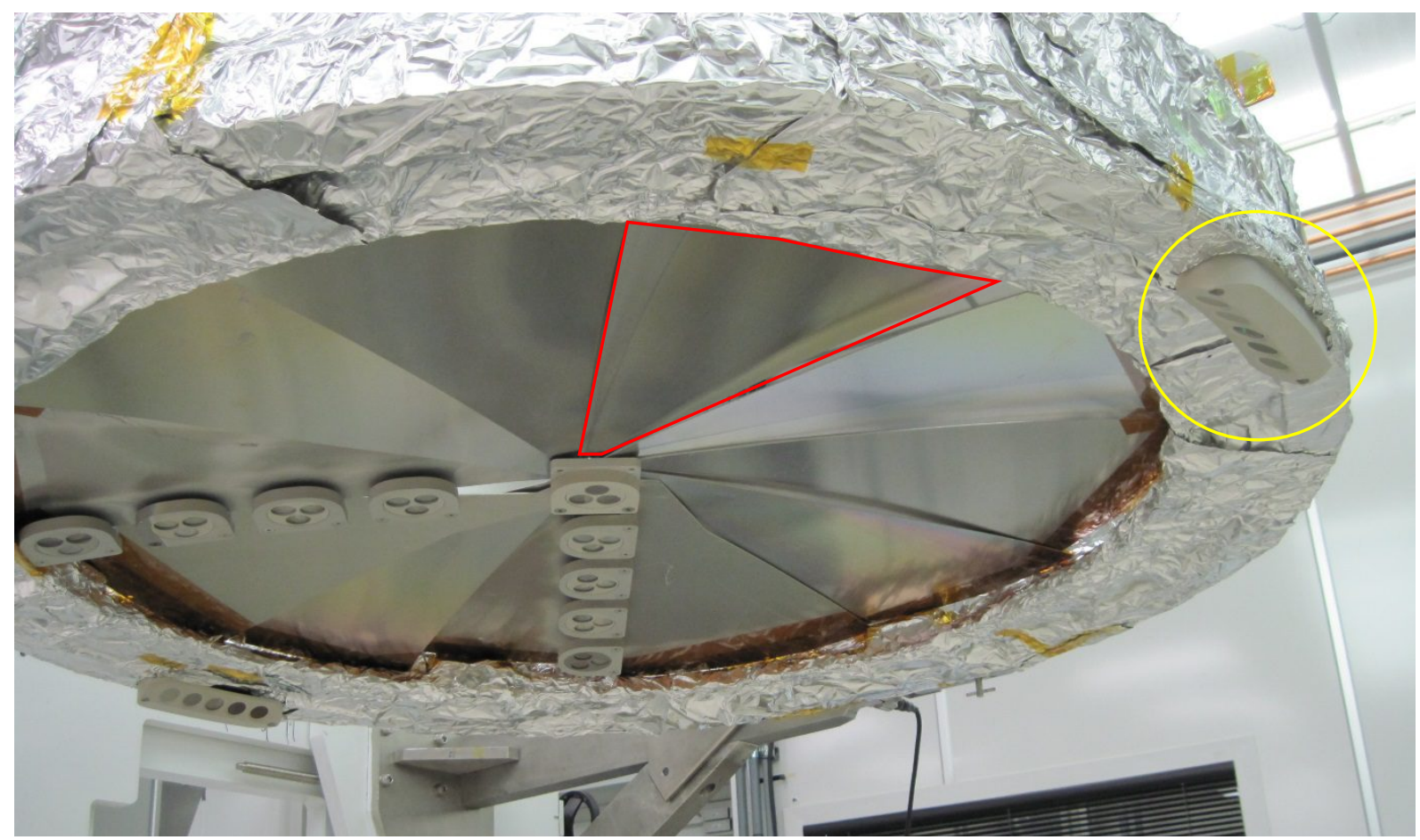

Figure 11. The C1 Side B surrogate installed into the coating ring with the surface wedges in place. One of the surface wedges is outlined in red. In all, 11 surface wedges were used to approximate the C1B surface. The small metal structure circled in yellow is one of the production witness holders where witnesses are held when a lens is being coated.

\section{METROLOGY}

During development and testing for each surface, witnesses were arrayed across the surrogate. Durability, stress, and spectroscopic metrology tests were conducted on witnesses at each radial position. Spectrophotometry measurements are made on a Shimadzu 3101 and Perkin Elmer Lambda 1050 instruments at the average angle of incidence for that radial position on the surface. When the lens surrogate structure is replaced by the lens then the performance of the coating on the lens surface can only be inferred from witnesses coated well outside the lens diameter as shown in Figure 11. To assure the lens coating is performing as tested, the lens surface is measured using a fiber spectrophotometer manufactured by Ocean Optics. Direct reflectance measurements are made at several points along a radius and those are used to show the actual performance of the lens surface across the radius. The witnesses are used to test for durability and provide a direct tie to previous test runs where the surrogate was coated.

\section{PERFORMANCE}

The specification for each surface is that the average transmission from $360 \mathrm{~nm}$ to $980 \mathrm{~nm}$ be $>98.5 \%$ and the minimum transmission at any point over the same band be $>98 \%$. This specification applies at all angles and for both substrate types - fused silica and N-BK7. Since each surface had a unique coating chamber set up, there were slight variations in the calibrations for each surface leading to variations in the surface to surface performance that would not be present in a fixed geometry. As referenced above, the chamber geometry was controlled to minimize the errors that set up changes could impart on the coating performance, but small variations in that set up geometry and manufacturing variability result in the transmission on each surface being slightly different. This is evident in Figure 12 where the transmission performance at the center of each of the 12 surfaces is plotted. The black dashed line is the single surface minimum requirement for the band average from $360 \mathrm{~nm}$ to $980 \mathrm{~nm}$. As shown in Table 2 the average across the $360 \mathrm{~nm}$ to $980 \mathrm{~nm}$ band exceeds $98.5 \%$ for all surfaces. The dash-dot line is the single surface minimum transmission allowed across the same band. 
In general, the transmission for all the surfaces is reasonably repeatable. As discussed in Section 4, at the blue end of the band, near $360 \mathrm{~nm}$, the variability due to the thin layers in the coating design become more apparent. Nine of the surfaces, represented by the 9 blue lines in Figure 12, have a transmission above 99\% for most of the band with performance well above the specifications and form a reasonably tight group. Three of the curves, C1A, C4A, and $\mathrm{ADC} 2 \mathrm{~B}$, (orange, red, and green respectively) have a transmission performance that is clearly "out of family" from the remaining 9 curves. We will discuss the coating geometry effect discovered on C4A that caused the rapid drop in transmission below $450 \mathrm{~nm}$, the high transmission above $750 \mathrm{~nm}$, and the shortened edge at $980 \mathrm{~nm}$ later in the paper. During the surrogate test runs, both $\mathrm{C} 1 \mathrm{~A}$ and $\mathrm{ADC} 2 \mathrm{~B}$ had a non-optimal high/low index ratio of the coating materials that created a small dip/valley in the transmission performance near $850 \mathrm{~nm}$. The surrogate performance, while not optimal, still met the spectral and repeatability requirements so the lens was coated. After coating the B side of the ADC2 lens the index ratio had increased slightly compared to the surrogate test runs, increasing the size of the transmission dip near $850 \mathrm{~nm}$ and causing the transmission to drop below $98 \%$ over a narrow region of the full band. The ADC2B band average is still $99 \%$. A similar occurrence with the A side of the $\mathrm{C} 1$ lens pushed the transmission very close to the minimum requirement but still stayed above $98 \%$. The minimum transmission value of $97.9 \%$ for $\mathrm{C} 1 \mathrm{~A}$ reported in Table 2 is from a single data point measured at $97.93 \%$ at $367 \mathrm{~nm}$.

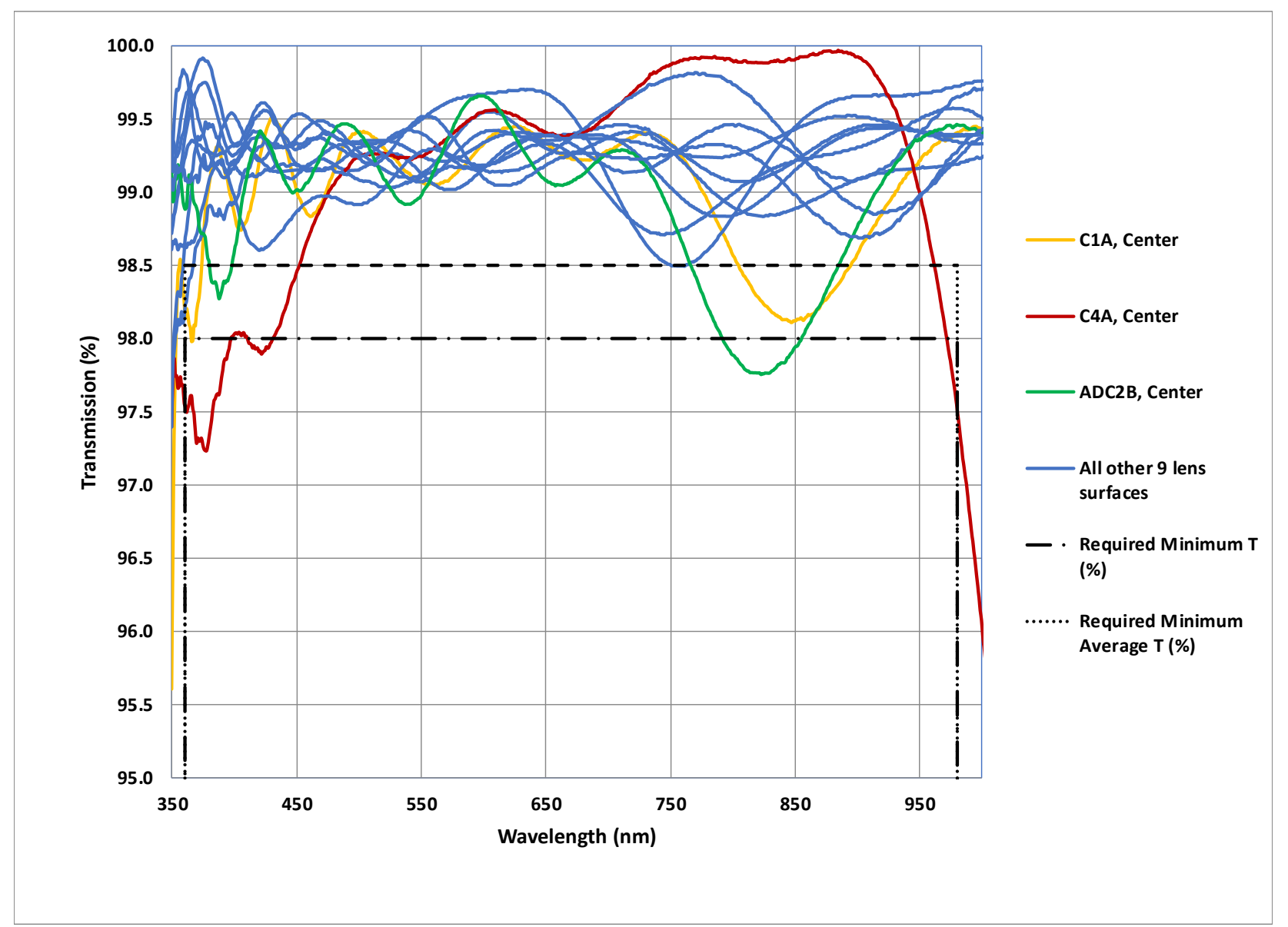

Figure 12. Transmission of the 12 lens surfaces at the center of each surface. The red, green and orange lines are for surfaces, $\mathrm{C} 4 \mathrm{~A}, \mathrm{ADC} 2 \mathrm{~B}$ and $\mathrm{C} 1 \mathrm{~A}$ respectively.

The transmission at the mid-radius and clear aperture radius of each lens is shown in Figures 13 and 14. The effect of the increasing angle of incidence and the non-uniformity across the radius is seen in these plots. The higher angle of incidence for some lens surfaces generally decreases the overall transmission which in turn gives a larger performance range of values. The thickness non-uniformity is very evident for the A side of C4. In Figure 5 it was shown the relative non-uniformity across the A side of $\mathrm{C} 4$ increases with radius until about mid-radius then rapidly drops to values less than 
the center thickness. That non-uniformity is the major cause of the $980 \mathrm{~nm}$ red edge of the C4A transmission moving slightly to longer wavelengths (red) at mid radius then sharply to shorter wavelengths (blue) at the edge of the clear aperture. Of note are the transmission valleys near $850 \mathrm{~nm}$ for A side of $\mathrm{C} 1$ (orange) and B side of ADC2 (green). The transmission at mid-radius, Figure 13, has increased near $850 \mathrm{~nm}$ compared to the center data. This is due to the same high/low index material thickness ratio having a small dependence on radial position. The trend continues in Figure 14 that shows the transmission of all surfaces at the clear aperture of each surface. By this radius, the thickness ratio has returned enough to nominal that the $\mathrm{B}$ side of $\mathrm{ADC} 2$ is completely within specification and the $\mathrm{A}$ side of $\mathrm{C} 1$ is much more "in family".

While the performance of individual lens surfaces is the basis of all the coating requirements, it is the system performance of all 6 lenses, 12 surfaces, that is the real end goal. The effect of the $850 \mathrm{~nm}$ transmission dip on the system performance is evident in the system transmission modeled in Figure 3. The transmission in the $800 \mathrm{~nm}$ to 850 $\mathrm{nm}$ region has a dip that is directly attributable to the dip on the C1A and ADC2B surfaces. Similarly, the rapid fall in clear aperture transmission from $900 \mathrm{~nm}$ to $980 \mathrm{~nm}$ in Figure 3 is directly attributable to the C4A transmission fall off beyond $900 \mathrm{~nm}$ shown in Figure 14. Comparing the impact of each lens surface performance allowed the DESI team to evaluate the lower performance of a single surface versus the risk of recoating to achieve higher performance. As each lens surface was coated it became apparent the transmission was generally significantly higher than required with a few exceptions, and system performance, while impacted, was still well above need and the risk of recoating a lens surface was not required.

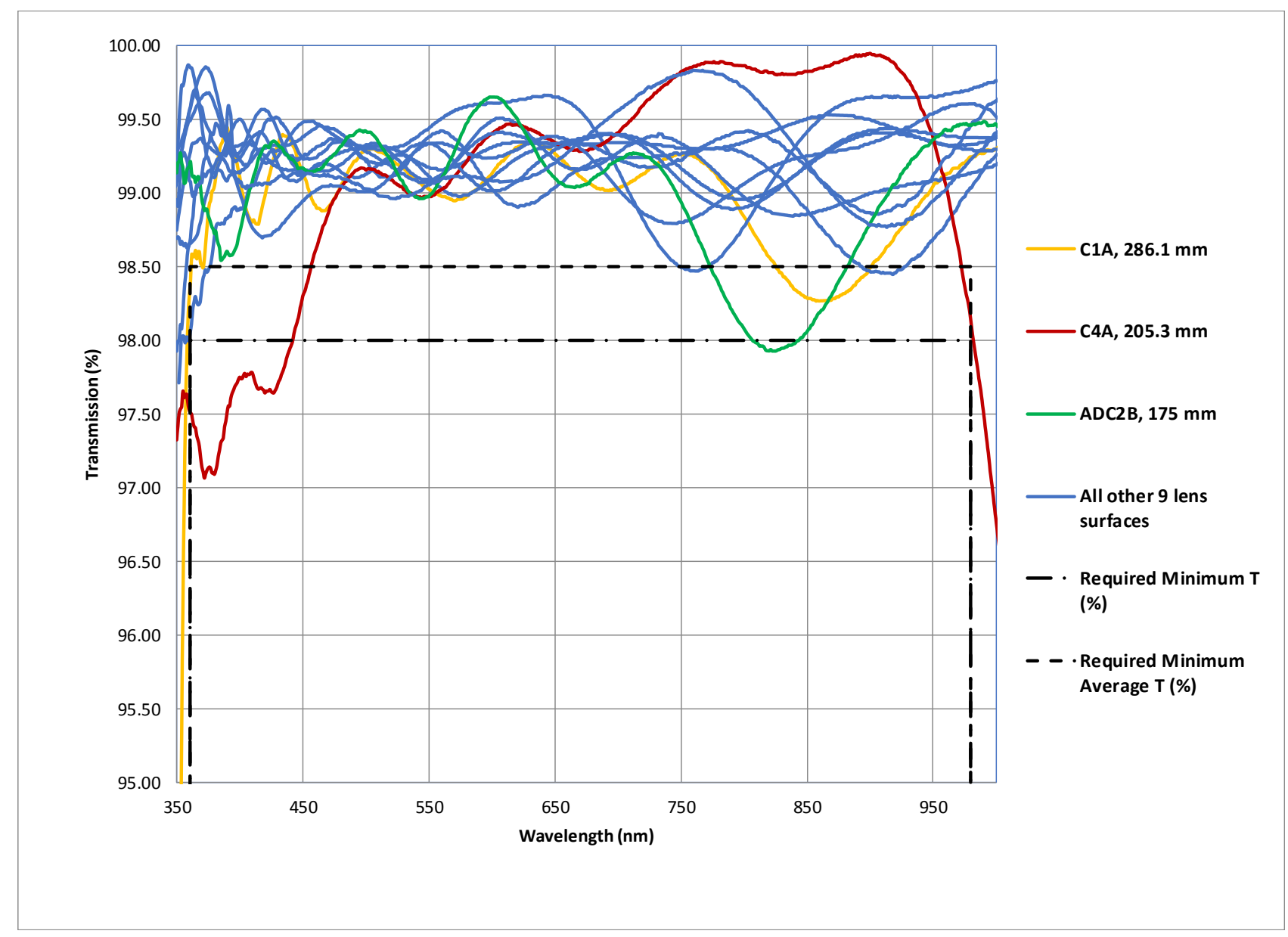

Figure 13. Transmission of the 12 lens surfaces at the mid-radius of each surface. The red, green and orange lines are for surfaces, $\mathrm{C} 4 \mathrm{~A}, \mathrm{ADC} 2 \mathrm{~B}$ and $\mathrm{C} 1 \mathrm{~A}$ respectively. 


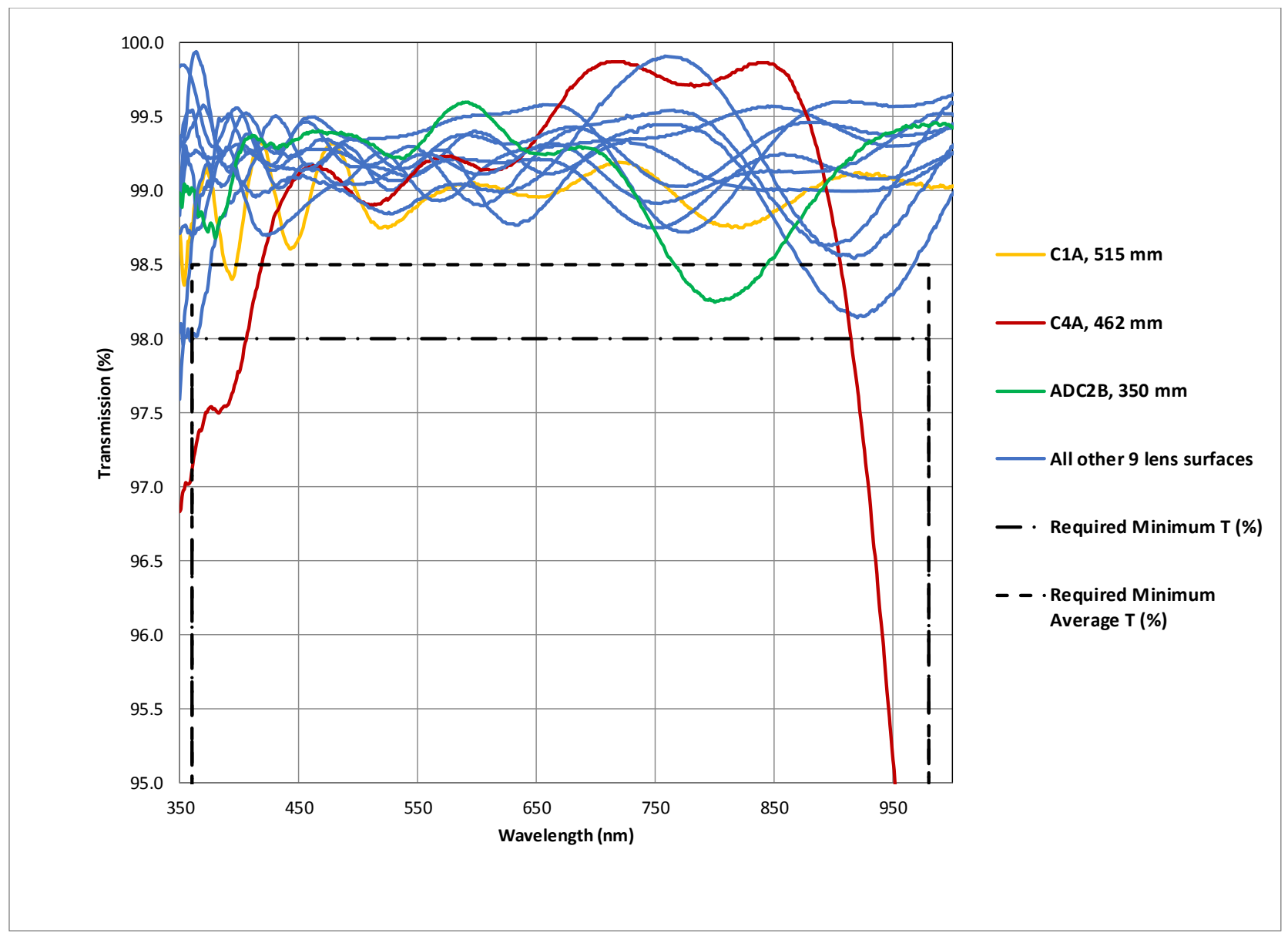

Figure 14. Transmission of the 12 lens surfaces at the clear aperture of each surface. The red, green and orange lines are for surfaces, $\mathrm{C} 4 \mathrm{~A}, \mathrm{ADC} 2 \mathrm{~B}$ and $\mathrm{C} 1 \mathrm{~A}$ respectively.

In Figures 12, 13, and 14 the performance of the A side of lens $\mathrm{C} 4$ is well out of family. The transmission drops off rapidly below $500 \mathrm{~nm}$ at all radii to below $98 \%$. Similarly, near $900 \mathrm{~nm}$ the transmission drops rapidly, with the outer radii showing the worst drop in performance. From the positioning of the long wavelength edge, it is clear that the centering of the coating is well short of the intended value. The tilt in the transmission curve below $500 \mathrm{~nm}$ is also indicative of coating materials having non-intended thickness values during the lens coating operations. Test coating runs for this lens surface using a surrogate designed to mimic the A side of Lens C4 had performances that would match very well with the rest of the lens surfaces plotted in the Figures 12, 13 and 14. Side A of lens C4 was the first lens surface to be coated. Measuring the witness samples after the lens coating was complete showed a significant shift in general centering toward shorter wavelengths where each material was affected differently causing the blue side tilt in the performance. Much analysis was completed and some additional metrology was developed following this coating run.

The early reliance on the witness samples coated with the lens proved insufficient to measure the actual performance of the lens surface after coating. This drove the move to acquire the fiber coupled spectrometer referenced earlier and measure each lens surface directly. The measurements made on the coated A side of lens C4 showed the centering and performance variation across the radius did not match the performance measured on the C4A surrogate used in the test runs immediately prior to coating the lens. The surrogates were designed to hold witnesses along a radius and match the curvature of the lens surface it is a surrogate for. It was simple and easily adaptable to differing shapes, but it did not mimic the entire surface, only a few slices of the surface. Much of the space between each radial arm of witnesses was left open, as can be inferred from Figures 9 and 10. In a large coating chamber, the path of the coating material from the deposition source to lens surface can be several mean free paths long, especially during higher pressure plasma-assisted deposition coating processes. In the Viavi coating chamber configuration for some of the lens surfaces the distance from 
deposition source to parts of the surface can be near $2 \mathrm{~m}$. By filling the open space between the witness holders of the coating surrogate using the surface wedges referred to in Section 5, we could replicate the performance measured on the coated A side of lens C4. The closed space surrogate adaptation is what was shown in Figure 11 previously. Lens surfaces coated subsequent to the A side of $\mathrm{C} 4$ matched much closer to the test coating runs using the surface surrogate done immediately prior to coating the lens surface.

While the transmission of the coated A side of lens $\mathrm{C} 4$ did not fully match program requirements the surrogate changes implemented after diagnosing and correcting the open space on the surrogates prevented a recurrence on the rest of the 11 lens surfaces. The superior performance on the rest of the lenses compensated for the A side of C4 lower transmission to assure system level performance was achieved.

\section{SUMMARY}

The DESI six lens optical system required overcoming several known challenges and one previously unencountered effect in order to successfully coat 12 different lens surfaces with a broadband anti-reflection coating. The tooling and handling practices were shaped by the size and weight of the lenses and specifics of the high temperature coating process. Use of Invar allowed common tooling for the two types of glass that safely supported the 6 lenses during coating operations and detailed procedures assured the safety of personnel and lenses during the multiple handling steps prior to and after coating. The coating design provided the ability to not use a masking system during coating and allowed for the wide angle of incidence range found on some of the lenses. The use of a fiber based spectrometer allowed direct reflectance measurements on the surfaces of the coated lenses providing non-interpreted measurements of the coating performance on each surface. While a coating process issue on one lens caused some lower than required transmission the higher performances on the rest of the lens surfaces allowed the 6-lens system performance to exceed requirements for both the $360 \mathrm{~nm}$ to $980 \mathrm{~nm}$ band transmission and the minimum allowed transmission. The final performance of the system will provide $\sim 90 \%$ transmission across the band which would which would have been $\sim 63 \%$ without the AR coating.

\section{ACKNOWLEDGEMENTS}

During this two year program, many people beyond the authors of this paper have directly influenced its successful completion. Whether it was a one day repair by a machinist, real time analysis in the Viavi Materials Characterization Lab, special set up durability testing or hallway discussions on whatever the problem was at the moment, it is all acknowledged and appreciated. During an especially critical point in the program, the meetings of a larger engineering team provided the pivotal discussions for explaining and correcting an unexpected shift in coating performance. In addition to the authors, other team members were Andre Anders and Eric Gullickson at Lawrence Berkeley Laboratory, Justin Wolfe at Lawrence Livermore National Laboratory and Rick Bradley, Marius Grigonis, Warren Hendricks, Karen Hendrix, Mark Kozlowski, Chris Piazzo, Markus Tilsch, and Fred Van Milligen at Viavi Solutions.

This research is supported by the Director, Office of Science, Office of High Energy Physics of the U.S. Department of Energy under Contract No. DE-AC02-05CH1123, and by the National Energy Research Scientific Computing Center, a DOE Office of Science User Facility under the same contract; additional support for DESI is provided by the U.S. National Science Foundation, Division of Astronomical Sciences under Contract No. AST-0950945 to the National Optical Astronomy Observatory; the Science and Technologies Facilities Council of the United Kingdom; the Gordon and Betty Moore Foundation; the Heising-Simons Foundation; the National Council of Science and Technology of Mexico, and by the DESI Member Institutions. The authors are honored to be permitted to conduct astronomical research on Iolkam Du'ag (Kitt Peak), a mountain with particular significance to the Tohono O'odham Nation.

\section{REFERENCES}

[1] Macleod, H. A., [Thin-Film Optical Filters], Macmillan Publishing Company, New York, (1986).

[2] Thelen, A., [Design of Optical Interference Coatings], McGraw-Hill Book Company, New York, (1989).

[3] Willey, R. R., "Further guidance for broadband antireflection design," Applied Optics 50(9), 274-278 (2011). 
[4] Schallenberg, U. B., “Antireflection designs concepts with equivalent layers," Applied Optics 45(7), 1507-1514 (2006).

[5] Tikhonravov, M., Amotchkina, T. and Dobrowolski, J., "Estimation of the average residual reflectance of broadband antireflection coatings," Applied Optics 47(13), 124-130 (2008).

[6] Flaugher, B., Bebek, C., "The Dark Energy Spectroscopic Instrument (DESI)", Proc. SPIE 9147 (2014).

[7] Doel, P., Sholl, M. J., Liang, M., Brooks, D., Flaugher, B, Gutierrez, G., Kent, S., Lampton, M., Miller, T., Sprayberry, D., "The DESI wide field corrector optics," Proc. SPIE 9147 (2014). 